\title{
An exact soluble equation for the steady state probability distribution in a nonlinear system: application to the noise reduction in Raman Ring Laser
}

\author{
Van Cao Long • Khoa Doan Quoc
}

Received: 1 March 2011 / Accepted: 23 November 2011 / Published online: 13 December 2011

(C) The Author(s) 2011. This article is published with open access at Springerlink.com

\begin{abstract}
In this paper, we consider the model of a nonlinear system subjected simultaneously to a random telegraph noise and a white noise. By using the formulae of differentiation introduced by Shapiro and Loginov (Physica 91:563-574, 1978), an exact equation for the steady state probability distribution of fluctuation in this system is derived. As an example of its application, we calculate analytically the steady state probability distribution of fluctuation and show the existence of noise induced phase transitions (Wódkiewicz et al. in J. Opt. Soc. Am. 1:398-405, 1984a) in Raman Ring Laser. Moreover, we show explicitly the so-called noise reduction considered in Lewenstein and Rzążewski (Opt. Commun. 63:174-178, 1987) for this system. It follows that the Stokes output of this laser tends to the stabilization under influence of the broad-band telegraph pump. This phenomenon could be realized experimentally in a much easer manner than for the case of Gaussian pump, because the construction of the injected telegraph pump signal is much easer than in the case of Gaussian signal. The recent paper is an extended version of Doan and Van (1991).
\end{abstract}

Keywords Exact soluble stochastic equations · Noise reduction · Raman Ring-laser

\section{Introduction}

It is well-known that it is very difficult, sometimes even impossible, to obtain exact analytical solutions for stochastic differential equations in the general case of arbitrary probability properties, in particular when we have arbitrary spectral characteristics of the external noises. Only in some special cases, as for white noise or pre-gaussian noise, the problem is exactly soluble. In this paper we consider a nonlinear system in which two these noises exist. For the sake of simplicity we restrict ourselves to the case when the pre-gaussian noise is just one dichotomous (telegraph) noise. In Sect. 2 following Wódkiewicz (1983) the exact

V. Cao Long $(\varangle) \cdot$ K. Doan Quoc

Quantum Optics and Engineering Division, Institute of Physics, University of Zielona Góra,

U1. Prof. Szafrana 4a, 65-516 Zielona Góra, Poland

e-mail: vanlongcao@yahoo.com 
equation for the steady state distribution for such system will be derived by using "formulae of differentiation" given in Shapiro and Loginov (1978).

In a typical problem of quantum optics, we have the resonance interaction of several lasers with an atomic (molecular) system, where the laser lights are fluctuating in amplitude and phase. Because of the very complicated (in many cases even obscure) microscopic nature of all relevant relaxation mechanisms, we model the laser lights by classical time-dependent random processes. The dynamical equations involved in the problem become stochastic differential equations. As it was emphasized above, except for some special cases the problem of obtaining an exact solution of such stochastic equations is a very difficult task. One of the most useful stochastic models has been introduced by Wódkiewicz et al. (1984a,b), Eberly et al. (1984), Cao Long and Wódkiewicz (1986) which is based on the so-called pregaussian process. It is composed of a finite number of independent telegraph signals mentioned above. The strength of the pregaussian formalism derives from exact solubility of wide classes of the stochastic equations. It follows from Sect. 2 that even in the case of one telegraph noise one can obtain several interesting results. Using the equation derived there we will consider in Sect. 3 the noise induced phase transitions of the type introduced in Kitahara et al. (1980) for Raman Ring Laser using the stochastic equation given in Lewenstein and Rzążewski (1987). As a consequence we will obtain the so-called noise reduction in this system. This phenomenon has been signed by one of us sometimes ago in the paper Doan and Van Long (1991). Section 4 contains our conclusions.

\section{The steady state probability distribution of fluctuation in a nonlinear system under the influence of telegraph noise}

We consider the nonlinear system which is described by the following generalized Langevin stochastic equation:

$$
\frac{d x}{d t}=F(x, \alpha(t))+\beta(t) h(x)
$$

where $F(x, \alpha(t))$ is a arbitrary nonlinear function of the investigated dynamical variable $x$ and the random process $\alpha(t)$. This random process is a telegraph noise with properties:

$$
\langle\alpha(t)\rangle=0,\left\langle\alpha(t) \alpha\left(t^{\prime}\right)\right\rangle=\Delta^{2} \exp \left(-\gamma\left|t-t^{\prime}\right|\right),
$$

whereas $\beta(t)$ is the white noise, which is independent with $\alpha(t)$ and

$$
\left\langle\beta(t) \beta\left(t^{\prime}\right)\right\rangle=2 D \delta\left(t-t^{\prime}\right) .
$$

Using the method described in Sancho and Miguel (1983), Wódkiewicz (1983) for Eq. (2.1), we can obtain an exact general equation for the steady state probability distribution of the dynamical variable $x$, as we will see below.

Following Wódkiewicz (1983) we introduce the quantity $\rho$ of $x$ with

$$
\rho(\xi, t)=\delta(\xi-x(t)) .
$$

Then the stochastic average of $x$ can be written by the probability distribution $P(\xi, t)$ of $x$ :

$$
\langle x\rangle=\int d \xi \xi P(\xi, t) .
$$


$P(\xi, t)$ is expressed by a path integration with a functional measure given in Wódkiewicz (1983). From definition (2.4) and Eq. (2.1) we obtain the Liouville equation in the form:

$$
\frac{\partial \rho(\xi, t)}{\partial t}=-\frac{\partial}{\partial \xi}[F(\xi, \alpha(t))+\beta(t) h(\xi)] \rho(\xi, t) .
$$

It follows from definition that the telegraph noise $\alpha(t)$ can have two values $\Delta$ and $-\Delta$ (the dichotomous Markovian process). Then we have the formula Kliatskin (1980)

$$
F(\xi, \alpha(t))=f(\xi)+g(\xi) \alpha(t),
$$

where

$$
f(\xi)=(F(\xi, \Delta)+F(\xi,-\Delta)) / 2, g(\xi)=(F(\xi, \Delta)-F(\xi,-\Delta)) / 2 \Delta
$$

are the even and odd parts of the function $F(\xi, \alpha(t))$, respectively.

Taking the stochastic average of the Liouville equation (2.6) we obtain the Fokker-PlanckStratonovitch equation for $P(\xi, t)$ :

$$
\frac{\partial P(\xi, t)}{\partial t}=-\frac{\partial}{\partial \xi} f(\xi) P(\xi, t)-\frac{\partial}{\partial \xi} g(\xi) P_{1}(\xi, t)-\frac{\partial}{\partial \xi} h(\xi)\langle\beta(t) \rho(\xi, t)\rangle,
$$

with $P(\xi, t)=\langle\rho(\xi, t)\rangle, P_{1}(\xi, t)=\langle\alpha(t) \rho(\xi, t)\rangle$. Using the differential formula in Shapiro and Loginov (1978) leads to:

$$
\begin{aligned}
\frac{\partial P_{1}(\xi, t)}{\partial t}= & -\left(\gamma+\frac{\partial}{\partial \xi} f(\xi)\right) P_{1}(\xi, t) \\
& -\Delta^{2} \frac{\partial}{\partial \xi} g(\xi) P(\xi, t)-\frac{\partial}{\partial \xi} h(\xi)\langle\beta(t) \alpha(t) \rho(\xi, t)\rangle .
\end{aligned}
$$

As a consequence of the Novikov theorem (1965) we have:

$$
\begin{aligned}
& \langle\beta(t) \rho(\xi, t)\rangle=-D \frac{\partial}{\partial x} h(\xi) P(\xi, t), \\
& \langle\beta(t) \alpha(t) \rho(\xi, t)\rangle=-D \frac{\partial}{\partial \xi} h(\xi) P_{1}(\xi, t) .
\end{aligned}
$$

Inserting Eqs. (2.11) and (2.12) into Eqs. (2.9) and (2.10), we obtain the following system of differential equations

$$
\begin{aligned}
\frac{\partial P(\xi, t)}{\partial t}= & -\frac{\partial}{\partial \xi} f(\xi) P(\xi, t)-\frac{\partial}{\partial \xi} g(\xi) P_{1}(\xi, t)+D \frac{\partial}{\partial \xi} h(\xi) \frac{\partial}{\partial \xi} h(\xi) P(\xi, t), \\
\frac{\partial P_{1}(\xi, t)}{\partial t}= & -\left(\gamma \frac{\partial}{\partial \xi} f(\xi)\right) P_{1}(\xi, t) \\
& -\Delta^{2} \frac{\partial}{\partial \xi} g(\xi) P(\xi, t)+D \frac{\partial}{\partial \xi} h(\xi) \frac{\partial}{\partial \xi} h(\xi) P_{1}(\xi, t) .
\end{aligned}
$$

It follows from Eq. (2.13) that in the stationary regime we obtain

$$
J(\xi)=f(\xi) P_{s}(\xi)+g(\xi) P_{1 s}(\xi)-D h(\xi) \frac{\partial}{\partial \xi} h(\xi) P_{s}(\xi)=\text { const },
$$

where $J(\xi)$ is the statistical current. The formula (2.5) justifies the change $\xi$ into $x$ in Eq. (2.15). Then we have

$$
J(x)=f(x) P_{s}(x)+g(x) P_{1 s}(x)-D h(x) \frac{\partial}{\partial x} h(x) P_{s}(x)=\text { const } .
$$


In almost all interesting physically cases in quantum optics we have $x \geq 0$, because $x$ is usually the amplitude or the intensity of the electric (laser) field, so we specify the boundary condition $\left.J(x)\right|_{x=0}=0$. This means that the statistical current should vanish in the region which is not physically meaningful $($ const $=0)$. Inserting Eq. $\left(2.15^{\prime}\right)$ with const $=0$ into Eq. (2.14), we obtain finally a differential equation for $P_{S}(x)$ :

$$
\left\{\Delta^{2} \frac{d}{d x} g(x)+\left[\gamma+\frac{d}{d x} f(x)-D \frac{d}{d x} h(x) \frac{d}{d x} h(x)\right] \frac{D h(x) \frac{d}{d x} h(x)-f(x)}{g(x)}\right\} P_{S}(x)=0 .
$$

The Eq. (2.16) is the exact cubic differential equation, which is difficult to be solved in the general case, so we can only solve it exactly in some simple cases. If in the stochastic differential equation (2.1) the white noise is neglected, the Eq. (2.14) now becomes:

$$
\left\{\Delta^{2} \frac{d}{d x} g(x)-\left[\gamma+\frac{d}{d x} f(x)\right] \frac{f(x)}{g(x)}\right\} P_{s}(x)=0,
$$

which leads to the general solution

$$
P_{s}(x)=N\left[\frac{g(x)}{\Delta^{2} g(x)^{2}-f(x)^{2}}\right] \exp \left(-\frac{\gamma}{2} \int^{x}\left[\frac{1}{f\left(x^{\prime}\right)-\Delta g\left(x^{\prime}\right)}+\frac{1}{f\left(x^{\prime}\right)+\Delta g\left(x^{\prime}\right)}\right] d x^{\prime}\right) \text {. }
$$

This expression of the stationary probability density is exactly the same what has been obtained by Kitahara et al. (1980). The zeroes of $\Delta^{2} g(x)^{2}-f(x)^{2}$ constitute the boundaries of the support of $P_{S}(x)$. Let $x_{1}$ and $x_{2}$ stand for the zeroes of the functions $F_{1,2}(x)=$ $f(x) \mp \Delta g(x)$. Then $P_{s}(x)$ in the vicinities of $x_{1}$ and $x_{2}$ is estimated (Kitahara et al. 1980)

$$
P_{S}\left(x_{1,2}+\delta x\right) \propto|\delta x|^{-\gamma / 2 F_{1,2}^{\prime}\left(x_{1,2}\right)-1}
$$

for $F_{1}\left(x_{1}\right)=F_{2}\left(x_{2}\right)=0, F_{1}\left(x_{2}\right) \neq 0$ and $F_{2}\left(x_{1}\right) \neq 0$. The condition for the system to be deterministically stable is that $F_{1}^{\prime}\left(x_{1}\right)<0$ and $F_{2}^{\prime}\left(x_{2}\right)<0$. It thus follows from Eq. (2.19) that $P_{s}(x)$ is normalizable. Furthermore we conclude from Eq. (2.19) that the stationary probability density $P_{S}(x)$ behaves near the boundary $x_{1,2}$ as follows:

$$
\begin{aligned}
& P_{s}(x)=\infty \quad \text { if }-\frac{\gamma}{2 F_{1,2}^{\prime}\left(x_{1,2}\right)}-1<0, \\
& P_{S}(x)=0 \quad \text { and } P_{s}^{\prime}(x)=\infty \text { if } 0<-\frac{\gamma}{2 F_{1,2}^{\prime}\left(x_{1,2}\right)}-1<1, \\
& P_{S}(x)=0 \quad \text { and } P_{s}^{\prime}(x)=0 \text { if } 1<-\frac{\gamma}{2 F_{1,2}^{\prime}\left(x_{1,2}\right)}-1 .
\end{aligned}
$$

One can easily verify from (2.18) that the extrema $x_{0}$ of $P_{S}(x)$ obey the following equation:

$$
f\left(x_{0}\right)-\frac{\Delta^{2}}{\gamma} g\left(x_{0}\right) g^{\prime}\left(x_{0}\right)+\frac{2}{\gamma} f\left(x_{0}\right) f^{\prime}\left(x_{0}\right)-\frac{1}{\gamma} f\left(x_{0}\right)^{2} \frac{g^{\prime}\left(x_{0}\right)}{g\left(x_{0}\right)}=0,
$$

Provided that $g\left(x_{0}\right)\left\{\Delta^{2} g\left(x_{0}\right)^{2}-f\left(x_{0}\right)^{2}\right\} \neq 0$. These extrema are usually identified with the macroscopic steady states and give us further information on the shape of $P_{S}(x)$. Our intuition frequently tells us that external noises destroy different effects related to the behavior of the system, but this is true only for the case of linear systems. In the case of the nonlinear systems, we have sometimes a very interesting phenomenon, namely the so-called the noise 
reduction, when the stationary distribution shrinks for increasing value of some parameter involved in the problem. This effect has been considered in a single mode Raman laser in a ring cavity (Lewenstein and Rzążewski 1987), where for certain regime of relaxation constants characterizing this laser, it is possible to achieve an efficient action via stimulated Raman scattering using a colored chaotic pump: The broader is the band pump, the less fluctuating is the Stokes output! As it has been emphasized by the authors in Lewenstein and Rzążewski (1987), the nonlinear stochastic equation derived there is rather difficult to analyze and a further simplification has been made. In the next Section we will analyze this equation exactly without any simplification for the case of the dichotomous noise.

\section{Noise reduction in a Raman Ring Laser}

The lasers based on the stimulated Raman scattering are pumped usually by a coherent light source. A special type of these lasers is so called Raman Ring Laser (see for example Roos et al. 2003; Luo et al. 2010; Zamzuri et al. 2011). The following question is very interesting: It is possible to create an efficient laser action by using an incoherent pump. As it is emphasized in Teubel and Rzążewski (1989), this question not only has theoretical relevance, but also has practical meaning, namely some ring Raman lasers have low threshold intensity. One can predict that the laser action with the help of conventional thermal sources can be achieved. Thus Raman Ring Lasers with a noisy pump is worth to study.

A single mode Raman laser in a ring cavity is described by the following system of differential equations:

$$
\begin{aligned}
\frac{d E_{p}(t)}{d t} & =-E_{S}(t)-\lambda\left[E_{p}(t)-Y(t)\right] \\
\frac{d E_{S}(t)}{d t} & =E_{p}(t) Q^{*}(t)-\mu E_{S}(t) \\
\frac{d Q(t)}{d t} & =E_{p}(t) E_{s}^{*}(t)-v Q(t),
\end{aligned}
$$

where $Q(t)$ is the atomic polarization, $E_{p}(t)$ is the pump field, $E_{s}(t)$ is the Stokes field, $Y(t)$ is the injected pump inside the cavity, $1 / \lambda$ and $1 / \mu$ are the lifetimes of cavity eigenmodes corresponding to the Pump and Stokes fields respectively, $1 / v$ is the polarization dephasing time. If $Y(t)$ represents a colored light we have:

$$
\langle Y(t)\rangle=0,\left\langle Y(t) Y^{*}\left(t^{\prime}\right)\right\rangle=\Delta^{2} \exp \left(-\gamma\left|t-t^{\prime}\right|\right),
$$

where $1 / \gamma$ is the coherence time of the injected Pump. Under typical experimental conditions:

$$
\lambda \gg v, \mu, \gamma \text {. }
$$

Therefore Pump field may be eliminated adiabatically from (3.1). we restrict ourselves to the case that

$$
v \gg \mu, \gamma \text {. }
$$

Under the condition (3.4) $Q(t)$ can be eliminated adiabatically too. These eliminations with the notation $x=E_{s}^{*}(t) E_{s}(t)$ lead to the following stochastic equation:

$$
\frac{d x}{d t}=-2\left[\mu-\frac{v \lambda^{2}|Y(t)|^{2}}{(x+\nu \lambda)^{2}}\right] x .
$$


It has been emphasized in Lewenstein and Rzążewski (1987) that the stochastic equation (3.5) is rather difficult to solve and some further assumption should be proposed. In Teubel and Rzążewski (1989) a numerical approach has been introduced which is more powerful than the analytical tools used in Lewenstein and Rzążewski (1987). We show now that this equation is exactly solved for the telegraph noise. For this purpose we assume that $Y(t)=\frac{1}{\sqrt{2}}\left(Y_{1}+i Y_{1}\right)$, where $Y_{1}=Y_{0}+I_{t}, I_{t}$ is a telegraph noise, in which $I_{t}$ can receive two values $\Delta$ and $-\Delta$. Then we obtain the equation of the type

$$
\frac{d x}{d t}=f(x)+I_{t} g(x)
$$

with

$$
\begin{aligned}
& f(x)=-2 \mu x+\frac{2 v \lambda^{2}\left(Y_{0}^{2}+\Delta^{2}\right)}{(x+v \lambda)^{2}} x, \\
& g(x)=\frac{4 v \lambda^{2} Y_{0}}{(x+v \lambda)^{2}} x .
\end{aligned}
$$

Substituting Eqs. (3.6) and (3.7) into thw expression (2.18), we obtain the following stationary probability density,

$$
P_{S}(x)=N x^{\frac{\gamma v^{2} \lambda^{2}}{4 \mu}\left(\frac{1}{x_{1} x_{1}^{\prime}}+\frac{1}{x_{2} x_{2}^{\prime}}\right)-1}\left(H_{1}+H_{2}\right)
$$

where

$$
\begin{aligned}
& x_{1}=\lambda\left[\sqrt{\frac{v}{\mu}}\left|Y_{0}-\Delta\right|-v\right], x_{1}^{\prime}=-\lambda\left[\sqrt{\frac{v}{\mu}}\left|Y_{0}-\Delta\right|+v\right] \text {, } \\
& x_{2}=\lambda\left[\sqrt{\frac{\nu}{\mu}}\left|Y_{0}+\Delta\right|-v\right], x_{2}^{\prime}=-\lambda\left[\sqrt{\frac{\nu}{\mu}}\left|Y_{0}+\Delta\right|+v\right] \text {, } \\
& H_{1}=\frac{\left(x_{1}+\nu \lambda\right)^{2}}{4 \mu \Delta}\left|x-x_{1}\right|^{\frac{\gamma}{8 \mu}\left(1+\frac{\nu \lambda}{x_{1}}\right)-1}\left|x-x_{1}^{\prime}\right|^{\frac{\gamma}{8 \mu}\left(1+\frac{\nu \lambda}{x_{1}^{\prime}}\right)-1}\left|x-x_{2}\right|^{\frac{\gamma}{8 \mu}\left(1+\frac{\nu \lambda}{x_{2}}\right)}\left|x-x_{2}^{\prime}\right|^{\frac{\gamma}{8 \mu}\left(1+\frac{\nu \lambda}{x_{2}^{\prime}}\right)} \text {, } \\
& H_{2}=\frac{\left(x_{2}+\nu \lambda\right)^{2}}{4 \mu \Delta}\left|x-x_{1}\right|^{\frac{\gamma}{8 \mu}\left(1+\frac{\nu \lambda}{x_{1}}\right)}\left|x-x_{1}^{\prime}\right|^{\frac{\gamma}{8 \mu}\left(1+\frac{\nu \lambda}{x_{1}^{\prime}}\right)}\left|x-x_{2}\right|^{\frac{\gamma}{8 \mu}\left(1+\frac{\nu \lambda}{x_{2}}\right)-1}\left|x-x_{2}^{\prime}\right|^{\frac{\gamma}{8 \mu}\left(1+\frac{\nu \lambda}{x_{2}^{\prime}}\right)-1} \text {. }
\end{aligned}
$$

We restrict ourselves to the case $x_{2}>0$, because $x$ is the Stokes intensity. Then the support of $P_{S}(x)$ is given by the interval $U=\left[\max \left(0, x_{1}\right), x_{2}\right]$.

Let us now study the behaviour of the stationary probability density in the neighbourhood of the boundaries of $U$ by using Eq. (2.19). For the upper boundary $x=x_{2}$, we have: $P_{s}\left(x_{2}\right)=\infty$ if $x_{2}>\frac{\gamma v \lambda}{8 \mu-\gamma}, P_{s}\left(x_{2}\right)=0$ and $P_{s}^{\prime}\left(x_{2}\right)=\infty$ if $\frac{\gamma v \lambda}{16 \mu-\gamma}<x_{2}<\frac{\gamma \nu \lambda}{8 \mu-\gamma}$, $P_{s}\left(x_{2}\right)=0$ and $P_{s}^{\prime}\left(x_{2}\right)=0$ if $x_{2}<\frac{\gamma \nu \lambda}{16 \mu-\gamma}$. For $x_{1}>0$ the lower boundary is given by $x_{1}$ and we have: $P_{s}\left(x_{1}\right)=\infty$ if $x_{1}>\frac{\gamma \nu \lambda}{8 \mu-\gamma}, P_{s}\left(x_{1}\right)=0$ and $P_{s}^{\prime}\left(x_{1}\right)=\infty$ if $\frac{\gamma \nu \lambda}{16 \mu-\gamma}<x_{1}<\frac{\gamma \nu \lambda}{8 \mu-\gamma}$, $P_{s}\left(x_{1}\right)=0$ and $P_{s}^{\prime}\left(x_{1}\right)=0$ if $x_{1}<\frac{\gamma \nu \lambda}{16 \mu-\gamma}$. For $x_{1}<0$ the lower boundary is given by zero. Since $x=0$ is a steady state of the deterministic equation (3.5) for all values of $Y_{t}$, obviously $F_{1}(0)=F_{2}(0)=0$ for $x_{1}<0$ and hence Eq. (2.19) cannot be used to discuss the behavior of $P_{S}(x)$ near the lower boundary of the support $U$ for this case. Analyzing directly with the explicit expression (3.8) for $P_{S}(x)$, we obtain: $P_{S}(0)=\infty$ if $\left(\frac{1}{x_{1} x_{1}^{\prime}}+\frac{1}{x_{2} x_{2}^{\prime}}\right)<\frac{4 \mu}{\gamma v^{2} \lambda^{2}}$, $P_{S}(0)=0$ and $P_{s}^{\prime}(0)=\infty$ if $\frac{4 \mu}{\gamma \nu^{2} \lambda^{2}}<\left(\frac{1}{x_{1} x_{1}^{\prime}}+\frac{1}{x_{2} x_{2}^{\prime}}\right)<\frac{8 \mu}{\gamma \nu^{2} \lambda^{2}}, P_{S}(0)=0$ and $P_{s}^{\prime}(0)=0$ if $\left(\frac{1}{x_{1} x_{1}^{\prime}}+\frac{1}{x_{2} x_{2}^{\prime}}\right)>\frac{8 \mu}{\gamma v^{2} \lambda^{2}}$. 


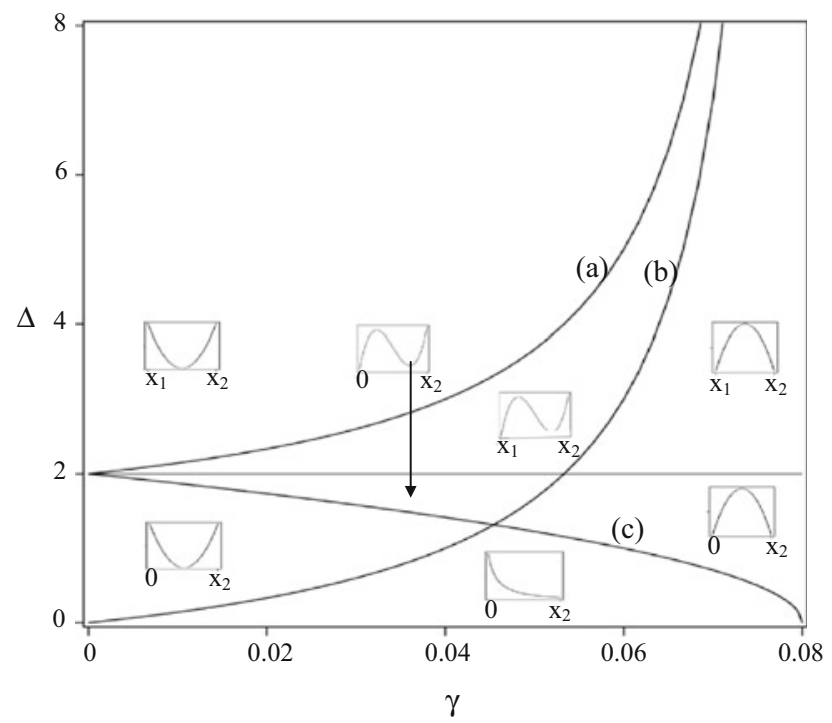

Fig. 1 Phase diagram for the steady state behavior of the model (3.5) as a function of intensity $\Delta$ and the coherence time of the injected pump $1 / \gamma$ of the colored noise, with lifetimes of cavity eigenmodes corresponding to the pump and Stokes fields $1 / \lambda=10^{-6}$ and $1 / \mu=10^{2}$, respectively. The polarization dephasing time is chosen as $1 / \nu=10^{-2}$. Curves $\mathbf{a}, \mathbf{b}$ and $\mathbf{c}$ are drawn correspondingly for: $x_{1}=\frac{\gamma \nu \lambda}{8 \mu-\gamma}, x_{2}=\frac{\gamma \nu \lambda}{8 \mu-\gamma}$ and $\left(\frac{1}{x_{1} x_{1}^{\prime}}+\frac{1}{x_{2} x_{2}^{\prime}}\right)=\frac{4 \mu}{\gamma v^{2} \lambda^{2}}$. In order not to burden the figure, the curves, where $P_{S}^{\prime}\left(x_{1,2}\right)$ changes from zero to infinity, have not been included. The shape of $P_{S}(x)$ (in $Y_{0}^{2}$ units), as concerns the extrema, is sketched for the different regions

Using Eq. (2.23), we see that the extrema of $P_{S}(x)$ obey the algebraic equation:

$$
\begin{gathered}
{[z-v \lambda]\left[\mu\left(\frac{6 \mu}{\gamma}-1\right) z^{5}-\frac{4 v \lambda \mu^{2}}{\gamma} z^{4}+v \lambda^{2}\left(1-\frac{4 \mu}{\gamma}\right)\left(Y_{0}^{2}+\Delta^{2}\right) z^{3}\right.} \\
\left.-\frac{2 v^{2} \lambda^{4}}{\gamma}\left(Y_{0}^{2}-\Delta^{2}\right)^{2} z+\frac{4 v^{3} \lambda^{5}}{\gamma}\left(Y_{0}^{2}-\Delta^{2}\right)^{2}\right]=0 .
\end{gathered}
$$

An analysis of the extrema of the function $P_{S}(x)$ and its behavior near the boundaries of the support $U$ permits us to construct a phase diagram of the steady state behavior of the system. According to different values of the parameters $\lambda, v, \mu, \gamma, \Delta, Y_{0}$ we have corresponding graphic forms of the function $P_{S}(x)$. We construct the phase diagram for the constant parameters $\lambda, v, \mu$, because we are interested in the influence of the pump width on $P_{s}(x)$. Phase diagram for the steady state behavior of the model (3.5) in the plane of $\gamma, \Delta$ is displayed in Fig. 1. Thus, the nonlinear system subjected to the telegraph (colored) noise may undergo phase transitions with the change of intensity and the correlation time of the noise. Following Kitahara et al. (1980) these transitions are called noise induced phase transitions.

In Fig. 2 we have plotted the steady probability distribution for a few values of $\gamma$ when the rest of the parameters are constant. In fact the distribution is narrower for increasing $\gamma$. Thus similar to the case of Gaussian colored noise, The Stokes output tends to be stabilized by the broad band pump, In our opinion, for the construction of a Raman laser with a broad-band pump which has been considered in Jones et al. (1985), Hefter et al. (1982), our results are of 
Fig. 2 The function $P_{S}(x)$ (in $Y_{0}^{2}$ units) which behaves near the boundaries $x_{1}$ and $x_{2}$ with the other values of the coherence time of the injected pump $1 / \gamma$. In which intensity of the noise $\Delta=8, \lambda=0.1, v=10^{-4}$, $\mu=10^{-7}$

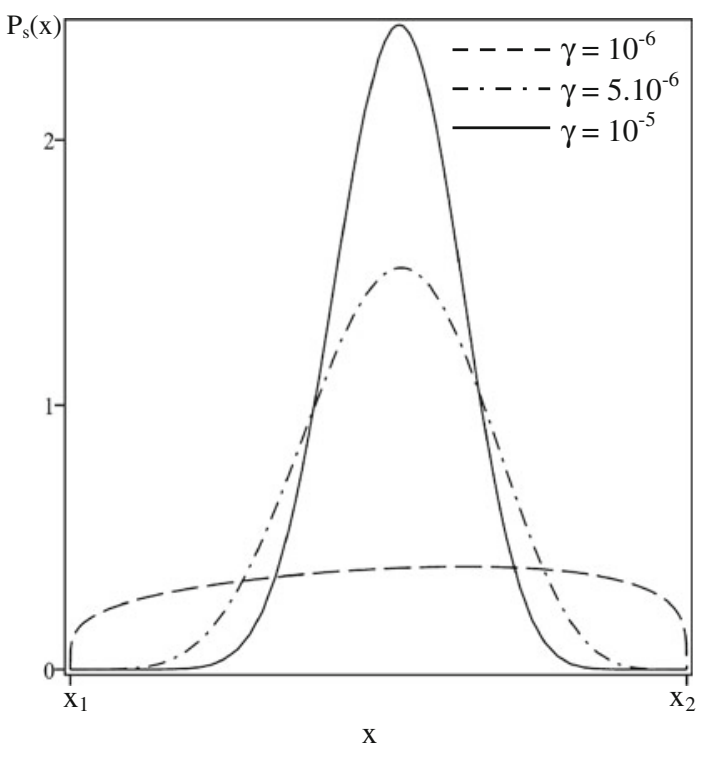

more direct experimental relevance than in the case of Gaussian noise, because the creation of the telegraph noise is much easer than Gaussian one. It is worth to note that a similar noise reduction has been considered for the lasers of an other type Hu and Peng (1998), where the effects of the finite pump bandwidth on the linewidth of a laser withour inversion have been presented. The authors have shown that under certain operating conditions the finite pump bandwidth does not lead to linewidth widening but to linewidth narrowing. This is not "surprising" as they have emphasized there: The same effect has been discovered almost eleven years earlier in Lewenstein and Rzążewski (1987).

\section{Conclusions}

In this paper we derived the exact equation for the steady probability distribution in the case of a nonlinear system influenced by two noises simultaneously: the telegraph noise and the white noise. As a byproduct we construct the phase diagram of Noise Induced Transition for the Raman ring-laser. It follows that the Stokes output of this laser tends to the stabilization under influence of the broad-band telegraph pump. This phenomenon could be realized experimentally in a much easer manner than for the case of Gaussian pump, because the construction of the injected telegraph pump signal is much easer than in the case of Gaussian signal.

Open Access This article is distributed under the terms of the Creative Commons Attribution Noncommercial License which permits any noncommercial use, distribution, and reproduction in any medium, provided the original author(s) and source are credited.

\section{References}

Cao Long, V., Wódkiewicz, K.: Multiphoton ionization in the presence of pre-Gaussian light. J. Phys. B 19, 1925-1933 (1986) 
Doan, B.B., Van, C.L.: Noise reduction in ring laser driven by a broad band pump. In: Internal Report IC/91/234. International Center of Theoretical Physics. Trieste, Italy (1991)

Eberly, J.H., Wódkiewicz, K., Shore, B.W.: Noise in strong laser-atom interactions: phase telegraph noise. Phys. Rev. A 30, 2381-2389 (1984)

Hefter, U. et al.: An optically pump supersonic lodine beam laser. Opt. Commun. 52, 330-335 (1982)

Hu, X.M., Peng, J.S.: Noise reduction via noise in a laser without inversion. J. Phys. B 31, L563-L569 (1998)

Jones, P.L. et al.: Optically pumped sodium-dimer supersonic-beam laser. Appl. Phys. Lett. 42, 222-224 (1985)

Kitahara, K., Horsthemke, W., Lefever, R., Inaba, Y.: Phase diagrams of noise induced transitions. Prog. Theor. Phys. 64, 1233-1247 (1980)

Kliatskin, W.I.: Stochastic Equations and Waves in Randomly Inhomogeneous Media. Nauka, Moscow (1980)

Lewenstein, M., Rzążewski, K.: Noise reduction in Raman ring-laser driven by chaotic pump. Opt. Commun. 63, 174-178 (1987)

Luo, Z., Yuan, X., Ye, W., Zeng, C., Ji, J.: Small-signal analysis of bidirectional operating characteristics in a Raman Ring Laser with external optical injections. Opt. Express 18, 19407-19412 (2010)

Novikov, E.A.: Functionals and the random force method in turbulence theory. Sov. Phys. JETP 20, 1290-1926 (1965)

Roos, P.A., Mei, L.S., Carsten, J.L.: Doppler-induced unidirectional operation of a continuous-wave Raman Ring Laser in H2. Appl. Opt. 42, 5517-5521 (2003)

Sancho, J.M., San Miguel, M.: Some results in the description of systems under the influence of dichotomous noise. Prog. Theor. Phys. 69, 1085-1090 (1983)

Shapiro, V.E., Loginov, V.M.: "Formulae of differentiation" and their use for solving stochastic equations. Physica 91, 563-574 (1978)

Teubel, A., Rzążewski, K.: Noise reduction in a Raman Ring Laser driven by a chaotic pump: numerical approach. J. Opt. Soc. Am. B 6, 550-553 (1989)

Wódkiewicz, K.: Langevin equations with colored noise in quantum optics. Acta Phys. Pol. A 63, 191-200 (1983)

Wódkiewicz, K., Shore, B.W., Eberly, J.H.: Pre-Gaussian noise in strong laser-atom interactions. J. Opt. Soc. Am. B 1, 398-405 (1984a)

Wódkiewicz, K., Shore, B.W., Eberly, J.H.: Noise in strong laser-atom interactions: frequency fluctuations and nonexponential correlations. Phys. Rev. A 30, 2390-2398 (1984b)

Zamzuri, A.K., Ismail, U.S., Al-Mansoori, M.H., Islam, M.S., Mahd, M.A.: All-optical wavelength conversion based on degenerate four-wave mixing in Raman Ring Laser. Laser Phys. Lett. 8, 62-65 (2011) 